\title{
TWO NEW WEYL-TYPE BOUNDS FOR THE DIRICHLET LAPLACIAN
}

\author{
LOTFI HERMI
}

\begin{abstract}
In this paper, we prove two new Weyl-type upper estimates for the eigenvalues of the Dirichlet Laplacian. As a consequence, we obtain the following lower bounds for its counting function. For $\lambda \geq \lambda_{1}$, one has

and

$$
N(\lambda)>\frac{2}{n+2} \frac{1}{H_{n}}\left(\lambda-\lambda_{1}\right)^{n / 2} \lambda_{1}^{-n / 2}
$$

$$
N(\lambda)>\left(\frac{n+2}{n+4}\right)^{n / 2} \frac{1}{H_{n}}\left(\lambda-(1+4 / n) \lambda_{1}\right)^{n / 2} \lambda_{1}^{-n / 2},
$$

where

$$
H_{n}=\frac{2 n}{j_{n / 2-1,1}^{2} J_{n / 2}^{2}\left(j_{n / 2-1,1}\right)}
$$

is a constant which depends on $n$, the dimension of the underlying space, and Bessel functions and their zeros.
\end{abstract}

\section{Four NeW Estimates}

Let $\Omega \subset \mathbb{R}^{n}$ be a bounded domain with piecewise smooth boundary. We are interested in bounds for the eigenvalues of the fixed and free membrane whose shape is assumed by $\Omega$. The first problem (also called the Dirichlet problem) is described by the equation

$$
\begin{aligned}
-\Delta u & =\lambda u \text { in } \Omega, \\
u & =0 \quad \text { on } \partial \Omega .
\end{aligned}
$$

Its eigenvalues, known to form a discrete countable family with no finite accumulation point (see [19] [25] for example), are denoted (counting multiplicity) by $0<\lambda_{1}<\lambda_{2} \leq \lambda_{3} \leq \ldots \leq \lambda_{k} \rightarrow \infty$. Its associated eigenfunctions, which form an orthonormal basis of real functions in $L^{2}(\Omega)$, are denoted by $u_{1}, u_{2}, u_{3}, \ldots$. The second problem (also called the Neumann problem [20]) is described by

$$
\begin{aligned}
-\Delta v & =\mu v \text { in } \Omega, \\
\frac{\partial v}{\partial n} & =0 \quad \text { on } \partial \Omega .
\end{aligned}
$$

Its eigenvalues, also discrete and countable, are denoted by $0=\mu_{1}<\mu_{2} \leq \mu_{3} \leq$ $\ldots \leq \mu_{k} \rightarrow \infty$. In this paper, we show the following.

Received by the editors April 15, 2004 and, in revised form, February 3, 2006.

2000 Mathematics Subject Classification. Primary 35P15; Secondary 47A75, 49R50, 58J50.

Key words and phrases. Eigenvalues of the Laplacian, Weyl asymptotics, Dirichlet problem, Neumann problem, Li-Yau bounds, Kröger bounds. 
Theorem 1.1. For $k \geq 1$, we have

$$
\lambda_{k+1}-\lambda_{1} \leq\left(1+\frac{n}{2}\right)^{2 / n} H_{n}^{2 / n} \lambda_{1} k^{2 / n}
$$

and the sharper, average-type, inequality

$$
\sum_{j=1}^{k}\left(\lambda_{j}-\lambda_{1}\right) \leq \frac{n}{n+2} H_{n}^{2 / n} \lambda_{1} k^{1+2 / n}
$$

where

$$
H_{n}=\frac{2 n}{j_{n / 2-1,1}^{2} J_{n / 2}^{2}\left(j_{n / 2-1,1}\right)} .
$$

As corollaries to these two inequalities, we prove (1.7) and (1.8). Here $J_{n}(x)$ and $j_{n, p}$ denote, respectively, the Bessel function of order $n$, and the $p$ th zero of this function (see [1]). The proof of this theorem is offered in Section 3. That (1.4) is sharper than (1.3) follows from left Riemann sum considerations (see Figure 1), namely

$$
\sum_{j=0}^{k-1} j^{2 / n}<\frac{k^{1+2 / n}}{1+2 / n} .
$$

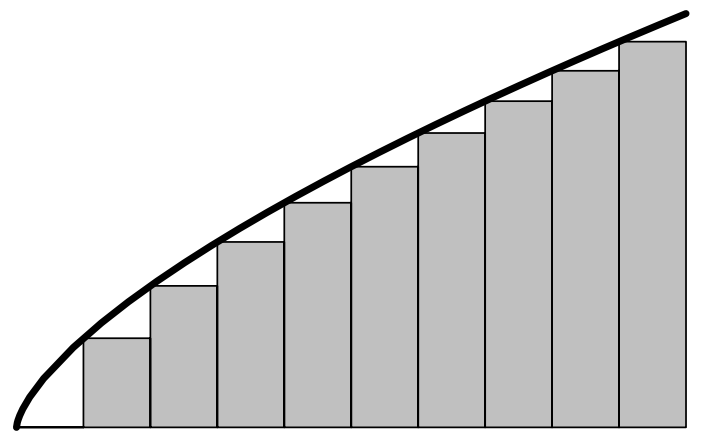

FiguRE 1. Left Riemann sums for $y=x^{2 / n}$, for $0 \leq x \leq k$.

To see this, we apply (1.3) to $j=0,1, \ldots, k-1$, then sum. We obtain

$$
\begin{aligned}
\sum_{j=1}^{k}\left(\lambda_{j}-\lambda_{1}\right) & \leq\left(1+\frac{n}{2}\right)^{2 / n} H_{n}^{2 / n} \lambda_{1}\left(\sum_{j=0}^{k-1} j^{2 / n}\right) \\
& <\left(1+\frac{n}{2}\right)^{2 / n} H_{n}^{2 / n} \lambda_{1} \frac{k^{1+2 / n}}{1+2 / n} .
\end{aligned}
$$

Inequality (1.4) is tighter since

$$
\left(1+\frac{n}{2}\right)^{2 / n}>1
$$

In fact, we have the following corollary. 
Corollary 1.2. For $k \geq 1$, the eigenvalues of the Dirichlet problem satisfy the estimate

$$
\sum_{j=2}^{k+1} \frac{1}{\lambda_{j}-\lambda_{1}} \geq \frac{n+2}{n} H_{n}^{-2 / n} \frac{1}{\lambda_{1}} \frac{k^{2}}{(k+1)^{1+2 / n}} .
$$

This of course follows from the Cauchy-Schwarz inequality

$$
\begin{aligned}
k^{2} & =\left(\sum_{j=2}^{k+1} \frac{1}{\sqrt{\lambda_{j}-\lambda_{1}}} \sqrt{\lambda_{j}-\lambda_{1}}\right)^{2} \\
& \leq \sum_{j=2}^{k+1} \frac{1}{\lambda_{j}-\lambda_{1}} \sum_{j=2}^{k+1}\left(\lambda_{j}-\lambda_{1}\right)
\end{aligned}
$$

and inequality (1.4). Another consequence of (1.4) and H. C. Yang's [52] (see also [3] [4], [15], [16], [17]) inequality

$$
\lambda_{k+1} \leq\left(1+\frac{4}{n}\right) \frac{1}{k} \sum_{j=1}^{k} \lambda_{j}
$$

is the bound

$$
\lambda_{k+1} \leq\left(1+\frac{4}{n}\right)\left(1+\frac{n}{n+2} H_{n}^{2 / n} k^{2 / n}\right) \lambda_{1}
$$

These types of inequalities follow the spirit of Weyl's asymptotic law, which states that

$$
\lambda_{k} \sim \frac{4 \pi^{2} k^{2 / n}}{\left(C_{n}|\Omega|\right)^{2 / n}} \text { as } k \rightarrow \infty,
$$

and

$$
\mu_{k+1} \sim \frac{4 \pi^{2} k^{2 / n}}{\left(C_{n}|\Omega|\right)^{2 / n}} \text { as } k \rightarrow \infty
$$

where $C_{n}=\frac{\pi^{n / 2}}{\Gamma(n / 2+1)}=$ volume of the unit $n$-ball, and $|\Omega|$ denotes the volume of $\Omega$. These formulas were proved by Weyl [51] in 1910 .

There is a beautiful exposé of the history of this problem in Kac's paper [30] (see also the equally entertaining paper [46]). Baltes and Hilf [18] trace the history of this type of asymptotic to Pockels (1891) (who proved the discreteness of the spectrum of the Dirichlet Laplacian [19]), Lord Rayleigh (1905), Sommerfeld (1910), and Lorentz (1910). Many asymptotics of this type were developed by Courant and Hilbert [25], Pleijel, and Minakshisundaram (see [18] for further insight and references).

In 1954, Pólya conjectured [42] that, for $k=1,2, \ldots$ (see also the series of papers [43] [44] [45])

$$
\mu_{k+1} \leq \frac{4 \pi^{2} k^{2 / n}}{\left(C_{n}|\Omega|\right)^{2 / n}} \leq \lambda_{k}
$$

He proved his conjecture for the case of tiling domains in a paper dedicated to Weyl in 1961 [43]. The restrictive conditions for the Neumann case in [43] (see also [45]) were relaxed, and the proof was refined and simplified by Kellner [31] "to Pólya's 
pleasure and satisfaction," reports Hersch (see p. 523 of [29]). In 1984, Urakawa [50] refined the Dirichlet bound to

$$
\lambda_{k} \geq \delta_{L}(\Omega)^{2 / n} \frac{4 \pi^{2} k^{2 / n}}{\left(C_{n}|\Omega|\right)^{2 / n}}
$$

where $\delta_{L}(\Omega)$ is the lattice packing density of $\Omega\left(\delta_{L}(\Omega)=1\right.$ for a tiling domain).

Also in 1954, Payne conjectured [39] that, independently of the Weyl term in $(1.9)$,

$$
\mu_{k+1} \leq \lambda_{k}
$$

This question was settled by Friedlander [28] in 1991 for domains with smooth boundaries. More recently, Friedlander's breakthrough was generalized for domains with non-smooth boundaries by Filonov [27]. On a different track, Li and Yau [37] proved that, for $k \geq 1$,

$$
\sum_{i=1}^{k} \lambda_{i} \geq \frac{n}{n+2} \frac{4 \pi^{2} k^{1+2 / n}}{\left(C_{n}|\Omega|\right)^{2 / n}},
$$

from which it obtains that

$$
\lambda_{k} \geq \frac{n}{n+2} \frac{4 \pi^{2} k^{2 / n}}{\left(C_{n}|\Omega|\right)^{2 / n}} .
$$

Inequalities (1.3), (1.4) and (1.8) can then be thought of as counterparts to these two inequalities of Li-Yau.

In 1992, Kröger [33] produced the Neumann parallels to the Li-Yau inequalities. For $k \geq 1$, he proved

$$
\sum_{i=1}^{k} \mu_{i} \leq \frac{n}{n+2} \frac{4 \pi^{2} k^{1+2 / n}}{\left(C_{n}|\Omega|\right)^{2 / n}}
$$

and

$$
\mu_{k+1} \leq\left(1+\frac{n}{2}\right)^{2 / n} \frac{4 \pi^{2} k^{2 / n}}{\left(C_{n}|\Omega|\right)^{2 / n}} .
$$

Notice that (1.15) implies that

$$
\begin{aligned}
\sum_{j=1}^{k} \mu_{j} \leq & \left(1+\frac{n}{2}\right)^{2 / n} \frac{4 \pi^{2}}{\left(C_{n}|\Omega|\right)^{2 / n}}\left(\sum_{j=0}^{k-1} j^{2 / n}\right) \\
& <\left(1+\frac{n}{2}\right)^{2 / n} \frac{4 \pi^{2}}{\left(C_{n}|\Omega|\right)^{2 / n}} \frac{k^{1+2 / n}}{1+2 / n}
\end{aligned}
$$

Again, we have used the left Riemann sum comparison (1.6) in these two inequalities. Kröger's inequality (1.14) is tighter, since

$$
\frac{(1+n / 2)^{2 / n}}{1+2 / n}>\frac{1}{1+2 / n}=\frac{n}{n+2} .
$$

Thus, the "averaged" version (namely (1.14)) of Kröger's two inequalities is sharper. 
Our bounds (1.3) and (1.8) are also related to the result of Ashbaugh and Benguria [9] who proved, for $m \geq 1$,

$$
\frac{\lambda_{2^{m}}}{\lambda_{1}} \leq\left(\frac{j_{n / 2,1}^{2}}{j_{n / 2-1,1}^{2}}\right)^{m}
$$

Of course, one cannot expect to fare better in the case of $m=1$ since this is another conjecture by Payne, Pólya, and Weinberger [40], [41] (herein referred to as PPW) which was settled by Ashbaugh and Benguria [5] (see also [6]) in 1991, namely

$$
\frac{\lambda_{2}}{\lambda_{1}} \leq \frac{j_{n / 2,1}^{2}}{j_{n / 2-1,1}^{2}}
$$

The ratio on the RHS of (1.17) is that for the two first eigenvalues of an $n$-ball. It has the asymptotic expansion [10]

$$
\frac{j_{n / 2,1}^{2}}{j_{n / 2-1,1}^{2}} \sim 1+\frac{4}{n}-\frac{4}{3} c_{1} \frac{2^{5 / 3}}{n^{5 / 3}}+\frac{12}{n^{2}}+\frac{4}{3}\left(c_{1}^{2}-2 c_{2}\right) \frac{2^{7 / 3}}{n^{7 / 3}}+O\left(n^{-8 / 3}\right),
$$

where $c_{1} \approx 1.8557571$ and $c_{2} \approx 1.033150$ (see [1]).

Payne, Pólya, and Weinberger [40], [41] (see also [9], [49]) proved the weaker form

$$
\lambda_{k+1}-\lambda_{k} \leq \frac{4}{n k} \sum_{j=1}^{k} \lambda_{j}
$$

from which one can infer that

$$
\frac{\lambda_{k+1}}{\lambda_{k}} \leq 1+4 / n
$$

and

$$
\frac{\lambda_{k}}{\lambda_{1}} \leq(1+4 / n)^{k-1}
$$

Note that (1.16) can be put in the form

$$
\frac{\lambda_{k}}{\lambda_{1}} \leq\left(\frac{j_{n / 2,1}^{2}}{j_{n / 2-1,1}^{2}}\right)^{\left[\frac{\log k}{\log 2}\right]}
$$

where $[x]$ stands for the integer part of $x$. This bound can be thought of as one of the form

$$
\left(\frac{j_{n / 2,1}^{2}}{j_{n / 2-1,1}^{2}}\right)^{\frac{\log k}{\log 2}}=k^{\frac{\log \left(j_{n / 2,1}^{2} / j_{n / 2-1,1}^{2}\right)}{\log 2}} .
$$

By virtue of the expansion (1.18), the power $\frac{\log \left(j_{n / 2,1}^{2} / j_{n / 2-1,1}^{2}\right)}{\log 2}$ has the asymptotic form

$$
\frac{1}{\log 2}\left(\frac{4}{n}-\frac{4}{3} c_{1} \frac{2^{5 / 3}}{n^{5 / 3}}+\frac{4}{n^{2}}+\frac{4}{3}\left(c_{1}^{2}-2 c_{2}\right) \frac{2^{7 / 3}}{n^{7 / 3}}\right)+O\left(n^{-8 / 3}\right),
$$


or

$$
\frac{\log \left(j_{n / 2,1}^{2} / j_{n / 2-1,1}^{2}\right)}{\log 2} \sim \frac{5.77078}{n}-6.10703 c_{1} \frac{1}{n^{5 / 3}}+O\left(\frac{1}{n^{2}}\right) .
$$

Thus, while tight at the bottom of the spectrum (viz. (1.17)), (1.16) does not capture the expected Weyl behavior of $k^{2 / n}$. Inequalities (1.3) and (1.8) remedy this.

The key to the new results is an observation by Ashbaugh and Benguria-the extent and limitations of which are discussed on p. 561 of [11]. If one identifies $\mu_{k+1}$ with $\lambda_{k+1}-\lambda_{1}$ and $|\Omega|^{-2 / n}$ with $\lambda_{1}$, then the RHS of the PPW inequality (1.17) can be seen as maximizing the ratio $\lambda_{2} / \lambda_{1}$ in the same vein as the quantity $C_{n}^{2 / n} p_{n / 2,1}^{2}$ maximizes $|\Omega|^{-2 / n} \mu_{2}$ for any domain $\Omega\left(p_{\nu, k}\right.$ denotes the $k$ th positive zero of the derivative of $x^{1-\nu} J_{\nu}(x)$ and $C_{n}$ is as defined above, i.e. the volume of the unit $n$-ball). The latter is a result of Szegö in 2 dimensions and Weinberger in $n$ dimensions. The maximum for both is assumed when $\Omega$ is an $n$-ball. The strategy of proof for both is similar, though the first is considerably more involved [5], [6]. This loose analogy can also be seen in comparing the methods of proof and results for

$$
\sum_{j=1}^{n}\left(\lambda_{j+1}-\lambda_{1}\right) \leq 4 \lambda_{1}
$$

and

$$
\sum_{j=1}^{n} \mu_{j+1} \leq n\left(\frac{C_{n}}{|\Omega|}\right)^{2 / n} p_{n / 2,1}^{2}
$$

both of which were proved by Ashbaugh and Benguria in [10] and [11]. Inequality (1.24) is the extension to $n$ dimensions of a result in [41]. (Note that (1.25) was proved with the further restriction that $\Omega$ is invariant with respect to $90^{\circ}$ rotations in the coordinate planes.) Our new inequality (1.4) can be viewed as an extension for $k \neq n$ of (1.24). (See Section 4 for a comparison with existing results.)

The loose correspondence can also be traced in the analogy between

$$
\sum_{k=1}^{n} \frac{1}{\lambda_{k+1}-\lambda_{1}} \geq \frac{2 j_{n / 2-1,1}^{2}+n(n-4)}{6 \lambda_{1}}>\frac{n^{2}}{4 \lambda_{1}}
$$

and

$$
\sum_{k=1}^{n} \frac{1}{\mu_{k+1}} \geq \frac{n\left(\frac{|\Omega|}{C_{n}}\right)^{2 / n}}{p_{n / 2,1}^{2}}
$$

Both of these bounds are also results found in [10] and [11] (with (1.27) also true under rotational symmetry of the base domain $\Omega$ ). Inequality $(1.26)$ is an extension and improvement of earlier results of Chiti [24]. The $n^{2} / 4 \lambda_{1}$ term in (1.26) is what corresponds to (1.24) via the "usual Cauchy-Schwarz connection" (viz. the proof of Corollary 1.2). On the other hand, there is also a conjectured inequality, from which, if proved, (1.26) would follow via the Cauchy-Schwarz argument. That 
inequality would be (1.24), but with its RHS replaced by

$$
\frac{6 n^{2} \lambda_{1}}{2 j_{n / 2-1,1}^{2}+n(n-4)} \text {. }
$$

Our new inequality (1.7) can be viewed as an extension, for $k \neq n$, of the Ashbaugh-Benguria-Chiti inequality (1.26).

We complete this section by giving the asymptotic expansions for the coefficients appearing in (1.3), and (1.8) (see [10] and [34] for similar estimates):

$$
\left(1+\frac{n}{2}\right)^{2 / n} H_{n}^{2 / n} \sim 1+\frac{2}{3 n} \log \frac{4 n^{4}}{b_{0}^{6}}+2^{8 / 3}\left(b_{1}-c_{1}\right)\left(\frac{1}{n}\right)^{5 / 3}+O\left(\frac{1}{n}\right)^{2} .
$$

In the case of (1.8), the expansion reads

$$
\begin{aligned}
&\left(1+\frac{4}{n}\right) \frac{n}{n+2} \quad H_{n}^{2 / n} \sim 1+\frac{2}{3 n}\left(3+\log \frac{32 n}{b_{0}^{6}}\right) \\
&+2^{8 / 3}\left(b_{1}-c_{1}\right)\left(\frac{1}{n}\right)^{5 / 3}+O\left(\frac{1}{n}\right)^{2} .
\end{aligned}
$$

Here $($ see $[1]) b_{0} \approx 1.1131028, b_{1} \approx 1.484606$ and $c_{1} \approx 1.8557571$.

\section{The COUnting FUnCtion}

One can motivate these inequalities in terms of the counting function,

$$
N(\lambda)=\sum_{\lambda_{k} \leq \lambda} 1=\sup _{\lambda_{k} \leq \lambda} k .
$$

Our Theorem 1.1 can then be restated.

Theorem 2.1. For $\lambda \geq \lambda_{1}$, we have the lower bounds

$$
N(\lambda)>\frac{2}{n+2} \frac{1}{H_{n}}\left(\lambda-\lambda_{1}\right)^{n / 2} \lambda_{1}^{-n / 2}
$$

and

$$
N(\lambda)>\left(\frac{n+2}{n+4}\right)^{n / 2} \frac{1}{H_{n}}\left(\lambda-(1+4 / n) \lambda_{1}\right)^{n / 2} \lambda_{1}^{-n / 2} .
$$

Remark. While (2.1) is a direct corollary to an earlier result of Laptev (see Cor. 4.4 in [35]) and Chiti's inequality (3.8) below (see [23], [24], [14]), (2.2) is new and in fact sharper. We refer the reader to the discussion in Section 4.

Proof. Inequalities (2.1) and (2.2) follow from (1.3) and (1.8), respectively. The proof for both is similar. We show (2.1) for illustration. Let $N(\lambda)=j$. Then, from the definition of $N(\lambda), \lambda_{j+1}>\lambda$. Inequality (1.3) then implies

$$
\lambda-\lambda_{1}<\left(1+\frac{n}{2}\right)^{2 / n} H_{n}^{2 / n} \lambda_{1} j^{2 / n} .
$$

Reversing, one gets (2.1).

Remark. Using an entirely different method, Safarov (see ineq. (2.9) of [48]) proved

$$
N(\lambda) \geq \frac{2}{n+2} e^{-1 / 4 \pi} L_{n}^{c l}\left(\lambda-\lambda_{1}\right)^{n / 2} \lambda_{1}^{-n / 2},
$$

where $L_{n}^{c l}=C_{n} /(2 \pi)^{n}$. In Table 1 we have listed numerical values of the coefficients appearing in (2.2), (2.1), and (2.3). These inequalities complete bounds of the form 
TABLE 1. Comparison of the coefficients appearing in (2.2), (2.1), and (2.3).

\begin{tabular}{|c|c|c||c|}
\hline \hline$n$ & $\left(\frac{n+2}{n+4}\right)^{n / 2} \frac{1}{H_{n}}$ & $\frac{2}{n+2} \frac{1}{H_{n}}$ & $\frac{2}{n+2} e^{-1 / 4 \pi} L_{n}^{c l}$ \\
\hline 2 & 0.259775 & 0.194831 & 0.036745 \\
3 & 0.201227 & 0.133333 & 0.062381 \\
4 & 0.167459 & 0.099235 & 0.000975 \\
5 & 0.145412 & 0.077874 & 0.000142 \\
6 & 0.129833 & 0.063395 & 0.000019 \\
7 & 0.118201 & 0.053193 & $2.5 \times 10^{-6}$ \\
\hline
\end{tabular}

$$
N(\lambda) \leq \tilde{K}_{n} \lambda^{n / 2}|\Omega|
$$

found in the works of Lieb [38] and Li-Yau [37] (see Laptev [35]). Of course, Weyl's asymptotic formula reads

$$
N(\lambda) \sim \frac{C_{n}|\Omega| \lambda^{n / 2}}{(2 \pi)^{n}}=L_{n}^{c l}|\Omega| \lambda^{n / 2},
$$

while the Pólya conjecture states

$$
N(\lambda) \leq L_{n}^{c l} \lambda^{n / 2}|\Omega| .
$$

The Li-Yau bounds can be reformulated as (see [35])

$$
N(\lambda) \leq\left(\frac{n+2}{n}\right)^{n / 2} L_{n}^{c l} \lambda^{n / 2}|\Omega| .
$$

In the same spirit, one should note F. Berezin's inequality [21] (see [47])

$$
\int_{0}^{\lambda} N(\mu) d \mu \leq \frac{2}{n+2} L_{n}^{c l} \lambda^{n / 2+1}|\Omega| .
$$

Laptev [35] and Safarov [47] have noted that the Li-Yau bound (2.7) is a corollary of (2.8). Indeed, for $\theta>0$,

$$
N(\lambda) \leq \frac{1}{\theta \lambda} \int_{0}^{\lambda+\theta \lambda} N(\mu) d \mu \leq \frac{2(1+\theta)^{n / 2+1}}{(n+2) \theta} L_{n}^{c l}|\Omega| \lambda^{n / 2} .
$$

Li-Yau's bound follows by setting $\theta=2 / n$. As for the Ashbaugh-Benguria inequality (1.16), it can be reworked to appear in the following terms (see [9]): For $\lambda \geq \lambda_{1}$

$$
N(\lambda) \geq 2^{\left[\log \left(\lambda / \lambda_{1}\right) / \log \left(j_{n / 2,1}^{2} / j_{n / 2-1,1}^{2}\right)\right]} .
$$

Notice that the RHS of this inequality assumes the form

$$
\left(\frac{\lambda}{\lambda_{1}}\right)^{\frac{1}{\log _{2} j_{n / 2,1}^{2} / j_{n / 2-1,1}^{2}}}
$$

This allows one to restate $(2.10)$ (in view of (1.23)) as

$$
N(\lambda) \geq\left(\frac{\lambda}{\lambda_{1}}\right)^{n / 5.77078}
$$


In fact, for $\lambda \geq \lambda_{2}$, Ashbaugh and Benguria have the sharper bound [9]

$$
N(\lambda) \geq 2^{1+\left[\log \left(\lambda / \lambda_{2}\right) / \log \left(j_{n / 2,1}^{2} / j_{n / 2-1,1}^{2}\right)\right]}
$$

which, in view of the above considerations, reads as

$$
N(\lambda) \geq 2\left(\frac{\lambda}{\lambda_{2}}\right)^{n / 5.77078} .
$$

\section{Proof of Theorem 1.1}

We begin with the Rayleigh-Ritz estimate for $\lambda_{k+1}$,

$$
\lambda_{k+1} \leq \inf _{r \geq r_{0}} \frac{\int_{B_{r}} \int_{\Omega}|\nabla \phi|^{2} d \mathbf{x} d \mathbf{z}}{\int_{B_{r}} \int_{\Omega}|\phi|^{2} d \mathbf{x} d \mathbf{z}},
$$

where $B_{r}=$ a ball of radius $r \geq r_{0}$, and $r_{0}=H_{n}^{1 / n}(1+k)^{1 / n} \sqrt{\lambda_{1}}$. This characterization is suggested by considerations similar to [33]. In fact, the bulk of the arguments follow steps described there. The test function $\phi$ is required to satisfy

$$
\phi \perp u_{1}, u_{2}, \ldots, u_{k} .
$$

It is chosen to be of the form

$$
\phi=e^{i \mathbf{x} \cdot \mathbf{z}} u_{1}(\mathbf{x})-\sum_{j=1}^{k} a_{j}(\mathbf{z}) u_{j}(\mathbf{x}) .
$$

The orthogonality conditions lead to $a_{j}(\mathbf{z})=\int_{\Omega} u_{1} \overline{u_{j}} e^{i \mathbf{x} \cdot \mathbf{z}} d \mathbf{x}$. We calculate

$$
\begin{aligned}
\int_{\Omega}|\phi|^{2} & =\int_{\Omega}\left|u_{1}\right|^{2}-2 \sum_{j}\left|a_{j}\right|^{2}+\sum_{j, \ell} a_{j} \overline{a_{\ell}} \int_{\Omega} u_{j} \overline{u_{\ell}} \\
& =1-\sum_{j=1}^{k}\left|a_{j}\right|^{2}
\end{aligned}
$$

since $\int_{\Omega} u_{j} \overline{u_{\ell}}=\delta_{j \ell}$. One has

$$
\int_{\Omega}|\nabla \phi|^{2}=\frac{1}{2}\left(\int_{\Omega} \phi(-\Delta \bar{\phi})+\int_{\Omega}(-\Delta \phi) \bar{\phi}\right)
$$

since $\phi$ and $\bar{\phi}$ satisfy the Dirichlet boundary condition. We let $\phi_{0}=e^{i \mathbf{x} \cdot \mathbf{z}}$. Then,

$$
\begin{aligned}
-\Delta \phi & =\left(-\Delta \phi_{0}\right) u_{1}-2 \nabla \phi_{0} \cdot \nabla u_{1}+\lambda_{1} \phi_{0} u_{1}-\sum a_{j} \lambda_{j} u_{j} \\
& =\left(-\Delta \phi_{0}\right) u_{1}-2 \nabla \phi_{0} \cdot \nabla u_{1}+\lambda_{1}\left(\phi+\sum a_{j} u_{j}\right)-\sum a_{j} \lambda_{j} u_{j} \\
& =\left(-\Delta \phi_{0}\right) u_{1}-2 \nabla \phi_{0} \cdot \nabla u_{1}+\lambda_{1} \phi-\sum a_{j}\left(\lambda_{j}-\lambda_{1}\right) u_{j} .
\end{aligned}
$$

Similarly,

$$
-\Delta \bar{\phi}=\left(-\Delta \bar{\phi}_{0}\right) \bar{u}_{1}-2 \nabla \bar{\phi}_{0} \cdot \nabla \bar{u}_{1}+\lambda_{1} \bar{\phi}-\sum \bar{a}_{j}\left(\lambda_{j}-\lambda_{1}\right) \bar{u}_{j} .
$$

Therefore,

$$
\int_{\Omega}(-\Delta \phi) \bar{\phi}=\lambda_{1} \int_{\Omega}|\phi|^{2}+\int_{\Omega}\left(-\Delta \phi_{0} u_{1}-2 \nabla \phi_{0} \cdot \nabla u_{1}\right) \bar{\phi}-\sum a_{j}\left(\lambda_{j}-\lambda_{1}\right) \int_{\Omega} u_{j} \bar{\phi} .
$$


Orthogonality makes $\int_{\Omega} u_{j} \bar{\phi}=0$.

$$
\int_{\Omega}(-\Delta \phi) \bar{\phi}=\lambda_{1} \int_{\Omega}|\phi|^{2}+\int_{\Omega}\left(-\Delta \phi_{0} u_{1}-2 \nabla \phi_{0} \cdot \nabla u_{1}\right) \bar{\phi} .
$$

We now concentrate on the quantity $\int_{\Omega}\left(-\Delta \phi_{0} u_{1}-2 \nabla \phi_{0} \cdot \nabla u_{1}\right) \bar{\phi}$. It is equal to

$$
\int_{\Omega}\left(-\Delta \phi_{0} \bar{\phi}_{0}\left|u_{1}\right|^{2}-2 \nabla \phi_{0} \cdot \nabla u_{1} \bar{\phi}_{0} \bar{u}_{1}\right)-\sum \bar{a}_{j} \int_{\Omega}\left(-\Delta \phi_{0} u_{1}-2 \nabla \phi_{0} \cdot \nabla u_{1}\right) \bar{u}_{j} .
$$

Since

$$
\frac{\partial \phi_{0}}{\partial x_{\ell}}=i z_{\ell} \phi_{0}
$$

and

$$
-\Delta \phi_{0}=|\mathbf{z}|^{2} \phi_{0}
$$

it obtains that

$$
\begin{aligned}
\int_{\Omega}\left(-\Delta \phi_{0} u_{1}-2 \nabla \phi_{0} \cdot \nabla u_{1}\right) \bar{\phi} & =|\mathbf{z}|^{2} \int_{\Omega}\left|u_{1}\right|^{2}+2 i \sum_{\ell=1}^{n} z_{\ell} \int_{\Omega} \bar{u}_{1} \frac{\partial u_{1}}{\partial x_{\ell}} \\
& -\sum_{j=1}^{k} \bar{a}_{j} \int_{\Omega}\left(-\Delta \phi_{0} u_{1}-2 \nabla \phi_{0} \cdot \nabla u_{1}\right) \bar{u}_{j} .
\end{aligned}
$$

We now use the identity $-\Delta \phi_{0} u_{1}-2 \nabla \phi_{0} \cdot \nabla u_{1}=-\Delta\left(\phi_{0} u_{1}\right)+\phi_{0} \Delta u_{1}$ (and the Dirichlet boundary condition) to reduce the second integral to

$$
\int_{\Omega}\left(-\Delta \phi_{0} u_{1}-2 \nabla \phi_{0} \cdot \nabla u_{1}\right) \bar{u}_{j}=\left(\lambda_{j}-\lambda_{1}\right) a_{j} .
$$

Substituting this into (3.4) and ultimately in (3.3) we conclude (since $\int_{\Omega}\left|u_{1}\right|^{2}=1$ )

$$
\int_{\Omega}(-\Delta \phi) \bar{\phi}=\lambda_{1} \int_{\Omega}|\phi|^{2}+|\mathbf{z}|^{2}+2 i \sum_{\ell=1}^{n} z_{\ell} \int_{\Omega} \bar{u}_{1} \frac{\partial u_{1}}{\partial x_{\ell}}-\sum_{j=1}^{k}\left|a_{j}\right|^{2}\left(\lambda_{j}-\lambda_{1}\right) .
$$

The term $\int_{\Omega} \bar{u}_{1} \frac{\partial u_{1}}{\partial x_{\ell}}$ is of course real, since $u_{1}$ was assumed to be real. Conjugating, we obtain

$$
\int_{\Omega} \phi(-\Delta \bar{\phi})=\lambda_{1} \int_{\Omega}|\phi|^{2}+|\mathbf{z}|^{2}-2 i \sum_{\ell=1}^{n} z_{\ell} \int_{\Omega} \bar{u}_{1} \frac{\partial u_{1}}{\partial x_{\ell}}-\sum_{j=1}^{k}\left|a_{j}\right|^{2}\left(\lambda_{j}-\lambda_{1}\right)
$$

Substituting (3.2), (3.5), and (3.6) into the Rayleigh-Ritz ratio (3.1) yields

$$
\lambda_{k+1}-\lambda_{1} \leq \frac{\int_{B_{r}}\left(|\mathbf{z}|^{2}-\sum_{j=1}^{k}\left|a_{j}(\mathbf{z})\right|^{2}\left(\lambda_{j}-\lambda_{1}\right)\right) d \mathbf{z}}{\int_{B_{r}}\left(1-\sum_{j=1}^{k}\left|a_{j}(\mathbf{z})\right|^{2}\right) d \mathbf{z}}
$$

or

$$
\lambda_{k+1}-\lambda_{1} \leq \frac{\int_{B_{r}}|\mathbf{z}|^{2} d \mathbf{z}-\sum_{j=1}^{k}\left(\lambda_{j}-\lambda_{1}\right) \int_{B_{r}}\left|a_{j}(\mathbf{z})\right|^{2} d \mathbf{z}}{\left|B_{r}\right|-\sum_{j=1}^{k} \int_{B_{r}}\left|a_{j}(\mathbf{z})\right|^{2} d \mathbf{z}} .
$$

We are now ready for our second reduction. We rescale the Fourier coefficient $a_{j}(\mathbf{z})$ by defining

$$
\tilde{a}_{j}(\mathbf{z})=\frac{1}{(2 \pi)^{n / 2}} \int_{\Omega} u_{1} \overline{u_{j}} e^{i \mathbf{x} \cdot \mathbf{z}} d \mathbf{x}=\frac{a_{j}(\mathbf{z})}{(2 \pi)^{n / 2}} .
$$


By Parseval's identity

$$
\begin{aligned}
\frac{1}{(2 \pi)^{n}} \int_{B_{r}}\left|a_{j}(\mathbf{z})\right|^{2} d \mathbf{z} & =\int_{B_{r}}\left|\tilde{a}_{j}(\mathbf{z})\right|^{2} d \mathbf{z} \\
& \leq \int_{\mathbb{R}^{n}}\left|\tilde{a}_{j}(\mathbf{z})\right|^{2} d \mathbf{z} \\
& =\int_{\Omega}\left|u_{1}\right|^{2}\left|u_{j}\right|^{2} d \mathbf{x} \\
& \leq \operatorname{ess} \sup \left|u_{1}\right|^{2} \int_{\Omega}\left|u_{j}(\mathbf{x})\right|^{2} d \mathbf{x} .
\end{aligned}
$$

We now use the following result of Chiti [23] (see also [24], [14]):

$$
\operatorname{ess} \sup \left|u_{1}\right| \leq\left(\frac{\lambda_{1}}{\pi}\right)^{n / 4} \frac{2^{1-n / 2}}{\Gamma(n / 2)^{1 / 2} j_{n / 2-1,1} J_{n / 2}\left(j_{n / 2-1,1}\right)} .
$$

Therefore,

$$
0<\int_{B_{r}}\left|a_{j}(\mathbf{z})\right|^{2} d \mathbf{z} \leq \widetilde{C}_{n} \lambda_{1}^{n / 2}
$$

where

We note that

$$
\widetilde{C}_{n}=\frac{2^{2} \pi^{n / 2}}{\Gamma(n / 2) j_{n / 2-1,1}^{2} J_{n / 2}^{2}\left(j_{n / 2-1,1}\right)} .
$$

$$
\pi^{n / 2}=\frac{n C_{n} \Gamma(n / 2)}{2}
$$

$\left(C_{n}\right.$ is the volume of the unit $n$-ball). Moreover, the constant $H_{n}$ defined in (1.5) is given by

$$
H_{n}=\frac{\widetilde{C}_{n}}{C_{n}}
$$

Remark. Safarov obtained (2.3) using the following result of E. B. Davies [26]:

$$
\text { ess } \sup \left|u_{1}\right| \leq e^{1 / 8 \pi} \lambda_{1}^{n / 4} \text {. }
$$

Chiti's statement (3.8) is an isoperimetric inequality. It saturates when $\Omega$ is an $n$-ball. Note that $e^{1 / 8 \pi} \approx 1.04059$, while the constant in $(3.8)$ takes the values listed in Table 2.

TABLE 2. Values of the constant in Chiti's bound (3.8) as a function of the dimension $n$.

\begin{tabular}{|c|c|}
\hline \hline$n$ & Chiti bound \\
\hline 2 & 0.451909 \\
3 & 0.225079 \\
4 & 0.103129 \\
5 & 0.044409 \\
6 & 0.018199 \\
7 & 0.007157 \\
\hline
\end{tabular}


We now prove by induction the following lemma.

Lemma 3.1. For $r \geq r_{0}(k)=H_{n}^{1 / n}(1+k)^{1 / n} \sqrt{\lambda_{1}}, k \geq 1$,

$$
\lambda_{k+1}-\lambda_{1} \leq \frac{\frac{n}{n+2} C_{n} r^{n+2}-\widetilde{C}_{n} \lambda_{1}^{n / 2} \sum_{j=1}^{k}\left(\lambda_{j}-\lambda_{1}\right)}{C_{n} r^{n}-k \widetilde{C}_{n} \lambda_{1}^{n / 2}} .
$$

Proof. We first note that

$$
\int_{B_{r}}|\mathbf{z}|^{2} d \mathbf{z}=\frac{n}{n+2} C_{n} r^{n+2}
$$

while

$$
\left|B_{r}\right|=\int_{B_{r}} d \mathbf{z}=C_{n} r^{n}
$$

These two facts reduce (3.7) to

$$
\lambda_{k+1}-\lambda_{1} \leq \frac{\frac{n}{n+2} C_{n} r^{n+2}-\sum_{j=1}^{k}\left(\lambda_{j}-\lambda_{1}\right) \int_{B_{r}}\left|a_{j}(\mathbf{z})\right|^{2} d \mathbf{z}}{C_{n} r^{n}-\sum_{j=1}^{k} \int_{B_{r}}\left|a_{j}(\mathbf{z})\right|^{2} d \mathbf{z}} .
$$

For $k=1$

$$
\lambda_{2}-\lambda_{1} \leq \frac{\frac{n}{n+2} C_{n} r^{n+2}}{C_{n} r^{n}-\int_{B_{r}}\left|a_{1}(\mathbf{z})\right|^{2} d \mathbf{z}} .
$$

It then obtains by virtue of (3.9) that, for $r \geq r_{0}(1)=H_{n}^{1 / n} 2^{1 / n} \sqrt{\lambda}_{1}$ (this condition guarantees that the denominator is positive)

$$
\lambda_{2}-\lambda_{1} \leq \frac{\frac{n}{n+2} C_{n} r^{n+2}}{C_{n} r^{n}-\widetilde{C}_{n} \lambda_{1}^{n / 2}},
$$

as desired. Suppose now that, for $r \geq r_{0}(k-1)=H_{n}^{1 / n} k^{1 / n} \sqrt{\lambda_{1}}$,

$$
\lambda_{k}-\lambda_{1} \leq \frac{\frac{n}{n+2} C_{n} r^{n+2}-\widetilde{C}_{n} \lambda_{1}^{n / 2} \sum_{j=1}^{k-1}\left(\lambda_{j}-\lambda_{1}\right)}{C_{n} r^{n}-(k-1) \widetilde{C}_{n} \lambda_{1}^{n / 2}} .
$$

Then, this is also true for $r \geq r_{0}(k)$ as well (since $r_{0}(k)>r_{0}(k-1)$ ). This implies

$$
\lambda_{k}-\lambda_{1} \leq \frac{\frac{n}{n+2} C_{n} r^{n+2}-\widetilde{C}_{n} \lambda_{1}^{n / 2}\left(\sum_{j=1}^{k-1}\left(\lambda_{j}-\lambda_{1}\right)+\left(\lambda_{k}-\lambda_{1}\right)\right)}{C_{n} r^{n}-(k-1) \widetilde{C}_{n} \lambda_{1}^{n / 2}-\widetilde{C}_{n} \lambda_{1}^{n / 2}}
$$

or

$$
\lambda_{k}-\lambda_{1} \leq \frac{\frac{n}{n+2} C_{n} r^{n+2}-\widetilde{C}_{n} \lambda_{1}^{n / 2} \sum_{j=1}^{k}\left(\lambda_{j}-\lambda_{1}\right)}{C_{n} r^{n}-k \widetilde{C}_{n} \lambda_{1}^{n / 2}} .
$$

(We have used the equivalence $\alpha \leq \frac{A}{B} \Leftrightarrow \alpha \leq \frac{A-\alpha \beta}{B-\beta}$, for $B-\beta \geq 0$.)

We also notice that if $A_{j} \geq 0$, then

$$
\frac{\sum_{j=1}^{k} A_{j}\left(\lambda_{j}-\lambda_{1}\right)}{\sum_{j=1}^{k} A_{j}} \leq \lambda_{k}-\lambda_{1}
$$


Hence, by virtue of (3.14), and for $A_{j}=\widetilde{C}_{n} \lambda_{1}^{n / 2}-\int_{\Omega}\left|a_{j}(\mathbf{z})\right|^{2} d \mathbf{z}$,

$$
\begin{aligned}
& \frac{\sum_{j=1}^{k}\left(\lambda_{j}-\lambda_{1}\right)\left(\widetilde{C}_{n} \lambda_{1}^{n / 2}-\int_{\Omega}\left|a_{j}(\mathbf{z})\right|^{2} d \mathbf{z}\right)}{\sum_{j=1}^{k}\left(\widetilde{C}_{n} \lambda_{1}^{n / 2}-\int_{\Omega}\left|a_{j}(\mathbf{z})\right|^{2} d \mathbf{z}\right)} \\
& \leq \frac{\frac{n}{n+2} C_{n} r^{n+2}-\widetilde{C}_{n} \lambda_{1}^{n / 2} \sum_{j=1}^{k}\left(\lambda_{j}-\lambda_{1}\right)}{C_{n} r^{n}-k \widetilde{C}_{n} \lambda_{1}^{n / 2}} .
\end{aligned}
$$

For simplicity we let

$$
\begin{gathered}
A=\frac{n}{n+2} C_{n} r^{n+2}-\widetilde{C}_{n} \lambda_{1}^{n / 2} \sum_{j=1}^{k}\left(\lambda_{j}-\lambda_{1}\right), \\
B=C_{n} r^{n}-k \widetilde{C}_{n} \lambda_{1}^{n / 2}, \\
C=\sum_{j=1}^{k}\left(\lambda_{j}-\lambda_{1}\right)\left(\widetilde{C}_{n} \lambda_{1}^{n / 2}-\int_{\Omega}\left|a_{j}(\mathbf{z})\right|^{2} d \mathbf{z}\right)
\end{gathered}
$$

and

$$
D=\sum_{j=1}^{k}\left(\widetilde{C}_{n} \lambda_{1}^{n / 2}-\int_{\Omega}\left|a_{j}(\mathbf{z})\right|^{2} d \mathbf{z}\right)
$$

By (3.12),

$$
\lambda_{k+1}-\lambda_{1} \leq \frac{A+C}{B+D} .
$$

Combining (3.15) and (3.16) (since $A / B=\Lambda$ and $C / D \leq \Lambda$ imply $(A+C) /$ $(B+D) \leq \Lambda)$, one obtains $(3.11)$, and the proof of the lemma is now complete.

If we set $r=r_{0}(k)=\left(\frac{\widetilde{C}_{n}}{C_{n}}\right)^{1 / n}(1+k)^{1 / n} \sqrt{\lambda_{1}}$ in the statement of Lemma 3.1, we obtain (1.4) (the "averaged" version) in the form

$$
\sum_{j=1}^{k+1}\left(\lambda_{j}-\lambda_{1}\right) \leq \frac{n}{n+2}\left(\frac{\widetilde{C}_{n}}{C_{n}}\right)^{2 / n} \lambda_{1}(1+k)^{1+2 / n} .
$$

This choice amounts to setting

$$
C_{n} r^{n}-k \widetilde{C}_{n} \lambda_{1}^{n / 2}=\widetilde{C}_{n} \lambda_{1}^{n / 2} .
$$

If we drop the sum in (3.11) and let $r=\tilde{r}(k)=\left(\frac{\widetilde{C}_{n}}{C_{n}}\right)^{1 / n} k^{1 / n} \sqrt{\lambda_{1}}\left(1+\frac{n}{2}\right)^{1 / n}$ we are led to (1.3), namely,

$$
\lambda_{k+1}-\lambda_{1} \leq\left(1+\frac{n}{2}\right)^{2 / n}\left(\frac{\widetilde{C}_{n}}{C_{n}}\right)^{2 / n} \lambda_{1} k^{2 / n} .
$$

This choice amounts to making

$$
C_{n} r^{n}-k \widetilde{C}_{n} \lambda_{1}^{n / 2}=\frac{k n}{2} \widetilde{C}_{n} \lambda_{1}^{n / 2} .
$$

(Note that $\tilde{r}(k) \geq r_{0}(k)$ since $n \geq 2$.) 
TABLE 3. Bound for $\frac{\lambda_{2}}{\lambda_{1}}$ as a function of the dimension $n$.

\begin{tabular}{|c|c|c|c|c|}
\hline \hline$n$ & $(1.20)$ & $(1.4)$ & $(1.3)$ & $(1.17)$ \\
\hline 2 & 3 & 6.133 & 6.133 & 2.539 \\
3 & 2.333 & 4.962 & 4.832 & 2.046 \\
4 & 2 & 4.556 & 4.174 & 1.796 \\
5 & 1.8 & 4.171 & 3.777 & 1.645 \\
6 & 1.667 & 3.986 & 3.508 & 1.543 \\
7 & 1.571 & 3.856 & 3.314 & 1.470 \\
\hline
\end{tabular}

Remark. The case $k=1$ in Lemma 3.1 provides a class of bounds for $\lambda_{2}-\lambda_{1}$ for $r \geq r_{0}(1)$. The function

$$
\frac{\frac{n}{n+2} C_{n} r^{n+2}}{C_{n} r^{n}-\widetilde{C}_{n} \lambda_{1}^{n / 2}}
$$

is non-increasing for $2^{1 / n} H_{n}^{1 / n} \sqrt{\lambda_{1}} \leq r \leq(1+n / 2)^{1 / n} H_{n}^{1 / n} \sqrt{\lambda_{1}}$ and nondecreasing beyond. At $r_{0}(1)$, it assumes the value of

$$
2^{1+2 / n} \frac{n}{n+2} H_{n}^{2 / n} \lambda_{1} \text {. }
$$

Hence

$$
\frac{\lambda_{2}}{\lambda_{1}} \leq 1+2^{1+2 / n} \frac{n}{n+2} H_{n}^{2 / n} .
$$

At its minimum (viz. $\left.r=(1+n / 2)^{1 / n} H_{n}^{1 / n} \sqrt{\lambda_{1}}\right)$, it assumes the form

$$
\lambda_{2}-\lambda_{1} \leq\left(1+\frac{n}{2}\right)^{2 / n} H_{n}^{2 / n} \lambda_{1} .
$$

Note that (3.20) is just (1.3) for $k=1$. Both bounds (3.19) and (3.20) are not expected to fare better than the Ashbaugh-Benguria inequality (1.17)-the best constant of its type (see Table 3). In fact, the first has the asymptotic expansion

$$
1+2^{1+2 / n} \frac{n}{n+2} H_{n}^{2 / n} \sim 3+\frac{2.53636}{n}-\frac{4}{3}\left(\frac{1}{n} \ln \frac{1}{n}\right)-\frac{4.71333}{n^{5 / 3}}+O\left(\frac{1}{n^{2}}\right) .
$$

Expanding the second, it obtains (see (1.28) above)

$$
1+\left(1+\frac{n}{2}\right)^{2 / n} H_{n}^{2 / n} \sim 2+\frac{0.495591}{n}-\frac{8}{3 n} \ln \frac{1}{n}-\frac{2.35666}{n^{5 / 3}}+O\left(\frac{1}{n^{2}}\right) .
$$

\section{Comparison With eXisting Results}

Consider the convex function

$$
\phi_{\lambda}(t)=(\lambda-t)_{+}=\left\{\begin{array}{c}
\lambda-t, \text { if } t \leq \lambda, \\
0, \text { if } t \geq \lambda .
\end{array}\right.
$$

In [35], Laptev proved (see Theo. 4.1) that

$$
\sum_{j}\left(\lambda-\lambda_{j}\right)_{+} \geq\left(\lambda-\lambda_{1}\right)^{1+n / 2} L_{n}^{c l} \frac{2}{n+2} \tilde{u}_{1}^{-2}
$$


where $\tilde{u}_{1}=\operatorname{ess} \sup \left|u_{1}\right|$. When $\lambda=\lambda_{2}$, (4.1) reduces to (see Cor. 4.2 of [35])

$$
\lambda_{2}-\lambda_{1} \leq\left(L_{n}^{c l} \frac{2}{n+2}\right)^{-2 / n} \tilde{u}_{1}^{4 / n}
$$

Combining this with the isoperimetric inequality of Chiti (3.8) gives (3.20). Note that Chiti's inequality (3.8) can be put in the form

$$
\tilde{u}_{1}^{2} \leq H_{n} L_{n}^{c l} \lambda_{1}{ }^{n / 2} .
$$

For $\lambda \geq \lambda_{1}$, Laptev's result (4.1) can also be interpreted as (see Cor. 4.4 of [35])

$$
N(\lambda) \geq\left(\lambda-\lambda_{1}\right)^{1+n / 2} L_{n}^{c l} \frac{2}{n+2} \tilde{u}_{1}^{-2}
$$

which, when combined with Chiti's ineq. (4.3), results in the statement of (2.1) with the same universal constant $\frac{2}{n+2} \frac{1}{H_{n}}$.

To obtain (1.3) from Laptev's vantage point, set $\lambda=\lambda_{k+1}$ in (4.1) and observe that

$$
k\left(\lambda_{k+1}-\lambda_{1}\right) \geq \sum_{j=1}^{k}\left(\lambda-\lambda_{j}\right)
$$

Hence

$$
\lambda_{k+1}-\lambda_{1} \leq k^{2 / n}\left(L_{n}^{c l} \frac{2}{n+2}\right)^{-2 / n} \tilde{u}_{1}^{4 / n} .
$$

Again, bounding $\tilde{u}_{1}$ using Chiti's isoperimetric inequality (4.3) yields the statement (1.3). In fact, one can write (4.1) in the form

$$
k\left(\lambda_{k+1}-\bar{\lambda}\right) \geq\left(\lambda_{k+1}-\lambda_{1}\right)^{1+n / 2} L_{n}^{c l} \frac{2}{n+2} \tilde{u}_{1}^{-2}
$$

where $\bar{\lambda}=\sum_{j=1}^{k} \lambda_{j} / k$. Therefore, using (3.8),

$$
\left(1+\frac{n}{2}\right) H_{n} \lambda_{1}^{n / 2} k\left(\lambda_{k+1}-\bar{\lambda}\right) \geq\left(\lambda_{k+1}-\lambda_{1}\right)^{1+n / 2} .
$$

This is a class of less accessible Weyl-type universal upper bounds for $\lambda_{k+1}$ different from both (1.3) and (1.4), but in the same spirit. Indeed, Cor. 4.4 from [35] (and eventually the weaker inequality (1.3)) follows from Theo. 4.1 of [35] by applying the rather rough estimate $\left(\lambda-\lambda_{j}\right)_{+} \leq\left(\lambda-\lambda_{1}\right)_{+}$. Refining this coarse estimate, one can recover (1.4) from Laptev's bound (4.1). Starting with (4.1), one first introduces the Legendre transform $\mathcal{L}\{f\}(p)=\sup _{\lambda \geq 0}(p \lambda-f(\lambda))$. It is then clear (see, e.g., [36]) that

$$
\mathcal{L}\left\{\sum_{j}\left(\lambda-\lambda_{j}\right)_{+}\right\}(p)=(p-[p]) \lambda_{[p]+1}+\sum_{j=1}^{[p]} \lambda_{j},
$$

where $[p]$ designates the integer part of $p$. The Legendre transform of the right hand side of (4.1) is given by

$$
\mathcal{L}\left\{\left(\lambda-\lambda_{1}\right)^{1+n / 2} L_{n}^{c l} \frac{2}{n+2} \tilde{u}_{1}^{-2}\right\}(p)=\lambda_{1} p+\frac{n}{n+2} p^{1+2 / n} \tilde{u}_{1}^{-4 / n}\left(L_{n}^{c l}\right)^{-2 / n}
$$


Since $f(\lambda) \geq g(\lambda)$ for all $\lambda \geq 0$ implies $\mathcal{L}\{f\}(p) \leq \mathcal{L}\{f\}(p)$ for all $p \geq 0$, we have (setting $p=k$ )

$$
\sum_{j=1}^{k}\left(\lambda_{j}-\lambda_{1}\right) \leq \frac{n}{n+2} \tilde{u}_{1}^{4 / n}\left(L_{n}^{c l}\right)^{-2 / n} k^{1+2 / n} .
$$

Combining the latter inequality with Chiti's inequality (3.8) we obtain (1.4).

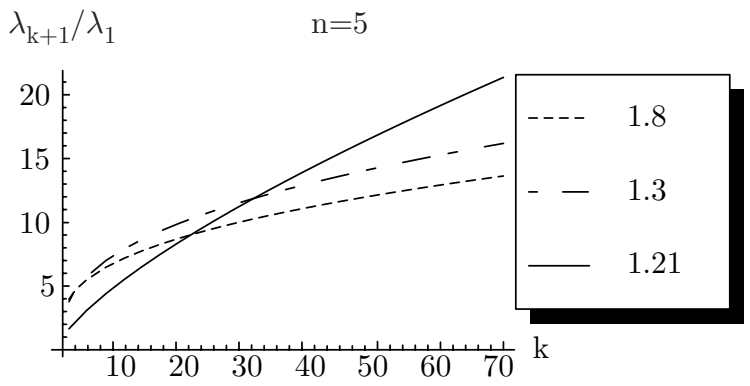

Figure 2. Comparison of new and old bounds.

Now, we turn to comparing these bounds. We claim that (1.8) is sharper than (1.3). To see this, we take the limit of the ratio of bounds as $k \rightarrow \infty$. This limit is equal to

$$
\frac{1+\frac{4}{n}}{\left(1+\frac{n}{2}\right)^{1+2 / n}}
$$

It is strictly less than 1 since $1+4 / n<1+n / 2<(1+n / 2)^{1+2 / n}$, for $n \geq 3$. This limit is equal to $3 / 4$ at $n=2$.

That both (1.3) and (1.8) are sharper than (1.21) (in the form (1.22)) follows from Krahn's second inequality [32] (see in particular ineq. (22) of [2])

$$
|\Omega|^{2 / n} \lambda_{2}>2^{2 / n} C_{n}^{2 / n} j_{n / 2-1,1}^{2} .
$$

For the unit ball in $\mathbb{R}^{n},|\Omega|=C_{n}$ and $\lambda_{2}=j_{n / 2,1}^{2}$. Therefore,

$$
\frac{j_{n / 2,1}^{2}}{j_{n / 2-1,1}^{2}}>2^{2 / n}
$$

or

$$
\frac{\log \left(j_{n / 2,1}^{2} / j_{n / 2-1,1}^{2}\right)}{\log 2}>\frac{2}{n}
$$

(see also (1.23) above). This is clearly displayed in Figure 2 where the AshbaughBenguria bound fares better to about $k=20$. The "averaged" bound (1.8) takes over and-at a latter stage-so does (1.3). Three tables are included in this paper which display this fact as well (see Tables 3-5). The new inequalities-both of which are disguised in earlier work of Laptev-cannot be expected to improve on existing bounds in the case of $\lambda_{2} / \lambda_{1}$. There is a competition (see Table 4) in the case of 
TABLE 4. Bound for $\frac{\lambda_{32}}{\lambda_{1}}$ as a function of the dimension $n$.

\begin{tabular}{|c|c|c|c|r|}
\hline \hline$n$ & $(1.20)$ & $(1.8)$ & $(1.3)$ & $(1.16)$ \\
\hline 2 & $6.177 \times 10^{14}$ & 122.334 & 160.112 & 105.46 \\
3 & $2.554 \times 10^{11}$ & 31.071 & 38.811 & 35.831 \\
4 & $2.147 \times 10^{9}$ & 15.606 & 18.675 & 18.707 \\
5 & $8.193 \times 10^{7}$ & 10.341 & 11.965 & 12.052 \\
6 & $7.539 \times 10^{6}$ & 7.870 & 8.878 & 8.758 \\
7 & $1.217 \times 10^{6}$ & 6.491 & 7.174 & 6.865 \\
\hline
\end{tabular}

TABLE 5. Bound for $\frac{\lambda_{128}}{\lambda_{1}}$ as a function of the dimension $n$.

\begin{tabular}{|c|c|c|c|r|}
\hline \hline$n$ & $(1.20)$ & $(1.8)$ & $(1.3)$ & $(1.16)$ \\
\hline 2 & $3.930 \times 10^{60}$ & 491.885 & 652.846 & 679.705 \\
3 & $5.408 \times 10^{46}$ & 75.911 & 97.808 & 149.957 \\
4 & $1.701 \times 10^{38}$ & 29.539 & 36.774 & 60.369 \\
5 & $2.628 \times 10^{32}$ & 16.814 & 20.2736 & 32.621 \\
6 & $1.496 \times 10^{28}$ & 11.593 & 11.5934 & 20.861 \\
7 & $8.500 \times 10^{24}$ & 8.917 & 8.917 & 14.836 \\
\hline
\end{tabular}

$\lambda_{32} / \lambda_{1}$ between (1.3) and (1.16) (already (1.8) is better than both for $n \geq 3$ ). In the case of $\lambda_{128} / \lambda_{1}$, both new bounds show considerable improvement (see Table $5)$.

\section{ACKNOWLEDGMENT}

The author offers his sincerest gratitude to Professors Lennie Friedlander and Mark Ashbaugh for comments and advice on drafts of this paper. The author also acknowledges the anonymous referee for insight on the route from (4.1) to (1.4).

\section{REFERENCES}

[1] M. Abramowitz and I. A. Stegun, editors, Handbook of Mathematical Functions, National Bureau of Standards Applied Mathematics Series, vol. 55, U.S. Government Printing Office, Washington, D.C., 1964. MR0167642 (29:4914)

[2] M. S. Ashbaugh, Open problems on eigenvalues of the Laplacian, in Analytic and geometric inequalities and applications, Th. M. Rassias and H. M. Srivastava, editors, Math. Appl., vol. 478, Kluwer Academic Publishers, Dordrecht, 1999, pp. 13-28. MR1785859 (2002j:35233)

[3] M. S. Ashbaugh, The universal eigenvalue bounds of Payne-Pólya-Weinberger, Hile-Protter, and H. C. Yang, in Spectral and inverse spectral theory (Goa, 2000), Proc. Indian Acad. Sci. Math. Sci. 112 (2002), 3-30. MR1894540 (2004c:35302)

[4] M. S. Ashbaugh, Isoperimetric and universal inequalities for eigenvalues, in Spectral Theory and Geometry, E. B. Davies and Yu. Safarov, editors, London Math. Soc. Lecture Series, vol. 273, Cambridge University Press, 1999, pp. 95-139. MR1736867 (2001a:35131)

[5] M. S. Ashbaugh and R. D. Benguria, Proof of the Payne-Pólya-Weinberger conjecture, Bull. Amer. Math. Soc. 25 (1991), 19-29. MR1085824 (91m:35173)

[6] M. S. Ashbaugh and R. D. Benguria, A sharp bound for the ratio of the first two eigenvalues of Dirichlet Laplacians and extensions, Ann. Math. 135 (1992), 601-628. MR1166646 (93d:35105) 
[7] M. S. Ashbaugh and R. D. Benguria, Isoperimetric inequalities for eigenvalue ratios, Partial Differential Equations of Elliptic Type, Cortona, 1992, A. Alvino, E. Fabes, and G. Talenti, editors, Symposia Mathematica, vol. 35, Cambridge University Press, Cambridge, 1994, pp. 1-36. MR1297771 (95h:35158)

[8] M. S. Ashbaugh and R. D. Benguria, Bounds for ratios of the first, second, and third membrane eigenvalues, in Nonlinear Problems in Applied Mathematics: In Honor of Ivar Stakgold on his Seventieth Birthday, T. S. Angell, L. Pamela Cook, R. E. Kleinman, and W. E. Olmstead, editors, Society for Industrial and Applied Mathematics, Philadelphia, 1996, pp. 30-42.

[9] M. S. Ashbaugh and R. D. Benguria, Bounds for ratios of eigenvalues of the Dirichlet Laplacian, Proc. Amer. Math. Soc. 121 (1994), 145-150. MR1186125 (94g:35157)

[10] M. S. Ashbaugh and R. D. Benguria, More bounds on eigenvalue ratios for Dirichlet Laplacians in $n$ dimensions, SIAM J. Math. Anal. 24 (1993), 1622-1651. MR1241161 (94i:35139)

[11] M. S. Ashbaugh and R. D. Benguria, Universal bounds for the low eigenvalues of Neumann Laplacians in $n$ dimensions, SIAM J. Math. Anal. 24 (1993), 557-570. MR1215424 (94b:35191)

[12] M. S. Ashbaugh and R. D. Benguria, Isoperimetric bound for $\lambda_{3} / \lambda_{2}$ for the membrane problem Duke Math. J. 63 (1991), 333-341. MR1115110 (92h:35165)

[13] M. S. Ashbaugh and R. D. Benguria, Isoperimetric bounds for higher eigenvalue ratios for the $n$-dimensional fixed membrane problem, Proc. Roy. Soc. Edinburgh Sect. A 123 (1993), 977-985. MR1263898 (95b:35162)

[14] M. S. Ashbaugh and L. Hermi, On extending the inequalities of Payne, Pólya, and Weinberger using spherical harmonics, to appear.

[15] M. S. Ashbaugh and L. Hermi, A unified approach to universal inequalities for eigenvalues of elliptic operators, Pacific J. Math. 217 (2004), 201-220. MR2109931 (2005k:35305)

[16] M. S. Ashbaugh and L. Hermi, On Harrell-Stubbe type inequalities for the discrete spectrum of a self-adjoint operator, preprint.

[17] M. S. Ashbaugh and L. Hermi, On Yang-type bounds for eigenvalues with applications to physical and geometric problems, preprint.

[18] H. Baltes and E. R. Hilf, Spectra of finite systems. A review of Weyl's problem: the eigenvalue distribution of the wave equation for finite domains and its applications on the physics of small systems, Bibliographisches Institut, Mannheim-Vienna-Zurich, 1976. MR0435624 $(55: 8582)$

[19] R. D. Benguria, Dirichlet Eigenvalue, in Encyclopaedia of Mathematics, Supplement vol. 3, M. Hazewinkel, Managing Editor, Kluwer Academic Publishers, 2001, pp. 130-132. MR1935796 (2003j:00009)

[20] R. D. Benguria, Neumann Eigenvalue, in Encyclopaedia of Mathematics, Supplement vol. 3, M. Hazewinkel, Managing Editor, Kluwer Academic Publishers, 2001, pp. 280-281. MR1935796 (2003j:00009)

[21] F. Berezin, Convariant and contravariant symbols of operators, Izv. Akad. Nauk SSSR 37 (1972), 1134-1167. [In Russian, English transl. in Math. USSR-Izv. 6 (1972), 1117-1151 (1973).] MR0350504 (50:2996)

[22] G. Chiti, A reverse Hölder inequality for the eigenfunctions of linear second order elliptic operators, J. Appl. Math. and Phys. (ZAMP) 33 (1982), 143-148. MR652928 (83i:35141)

[23] G. Chiti, An isoperimetric inequality for the eigenfunctions of linear second order elliptic operators, Boll. Un. Mat. Ital. (6) 1-A (1982), 145-151. MR652376 (83i:35140)

[24] G. Chiti, Inequalities for the first three membrane eigenvalues, Boll. Un. Mat. Ital. 18-A (1981), 144-148. MR607218 (82g:35087)

[25] R. Courant and D. Hilbert, Methoden der mathematischen Physik, vol. I, Springer Verlag, 1931. (English edition: Methods of Mathematical Physics, vol. I, Interscience, New York, 1953.) MR49:8778

[26] E. B. Davies, Heat Kernels and Spectral Theory, Cambridge Tracts in Mathematics 92, Cambridge University Press, Cambridge, 1989. MR990239 (90e:35123)

[27] N. Filonov, On an inequality for the eigenvalues of the Dirichlet and Neumann problems for the Laplace operator (Russian), Algebra i Analiz 16 (2004), 172-176. MR2068346 (2005f:35228) 
[28] L. Friedlander, Some inequalities between Dirichlet and Neumann eigenvalues, Arch. Rational Mech. Anal. 116 (1991), 153-160. MR1143438 (93h:35146)

[29] J. Hersch and G.-C. Rota, editors, George Pólya: Collected Papers, vol. III: Analysis, MIT Press, Cambridge, Massachusetts, 1984. MR758989 (85m:01108a)

[30] M. Kac, Can one hear the shape of a drum?, Amer. Math. Monthly 73 (1966), 1-23. MR0201237 (34:1121)

[31] R. Kellner, On a theorem of Pólya, Amer. Math. Monthly 73 (1966), 856-858. MR0200623 $(34: 514)$

[32] E. Krahn, Über Minimaleigenschaften der Kugel in drei und mehr Dimensionen, Acta Comm. Univ. Tartu (Dorpat) A9 (1926), 1-44. [English translation: Minimal properties of the sphere in three and more dimensions, in Edgar Krahn 1894-1961: A Centenary Volume, Ü. Lumiste and J. Peetre, editors, IOS Press, Amsterdam, 1994, Chapter 11, pp. 139-174.] MR1298188 (95d:01022)

[33] P. Kröger, Upper bounds for the Neumann eigenvalues on a bounded domain in Euclidean space, J. Funct. Anal. 106 (1992), 353-357. MR1165859 (93d:47091)

[34] R. Laugesen, Eigenvalues of the Laplacian on inhomogeneous membranes, Amer. J. Math. 120 (1998), 305-344. MR1613638 (99b:35161)

[35] A. Laptev, Dirichlet and Neumann eigenvalue problems on domains in Euclidean spaces, J. Funct. Anal. 151 (1997), 531-545. MR1491551 (99a:35027)

[36] A. Laptev and T. Weidl, Recent results on Lieb-Thirring inequalities, Journées "Équations aux Dérivées Partielles" (La Chapelle sur Erdre, 2000), Exp. No. XX, 14 pp., Univ. Nantes, Nantes, 2000. MR1775696 (2001j:81064)

[37] P. Li and S.-T. Yau, On the Schrödinger equation and the eigenvalue problem, Comm. Math. Phys. 88 (1983), 309-318. MR701919 (84k:58225)

[38] E. H. Lieb, The number of bound states of one-body Schroedinger operators and the Weyl problem, in Geometry of the Laplace operator (Proc. Sympos. Pure Math. Univ. Hawaii, Honolulu, Hawaii, 1979), Proc. Sympos. Pure Math. XXXVI, Amer. Math. Soc. Providence, R.I., 1980, pp. 241-252. MR573436 (82i:35134)

[39] L. E. Payne, Inequalities for eigenvalues of membranes and plates, J. Rational Mech. Anal. 4 (1955), 517-529. MR0070834 (17:42a)

[40] L. E. Payne, G. Pólya, and H. F. Weinberger, Sur le quotient de deux fréquences propres consécutives, Comptes Rendus Acad. Sci. Paris 241 (1955), 917-919 (reprinted as pp. 410-412 of [29] with comments by J. Hersch on p. 518). MR0073046 (17:372d)

[41] L. E. Payne, G. Pólya, and H. F. Weinberger, On the ratio of consecutive eigenvalues, J. Math. and Phys. 35 (1956), 289-298 (reprinted as pp. 420-429 of [29] with comments by J. Hersch on p. 521). MR0084696 (18:905c)

[42] G. Pólya, Mathematics and Plausible Reasoning, Vol. II. Princeton Univ. Press, Princeton, N.J., 1954, pp. 51-53. MR92a:00028b

[43] G. Pólya, On the eigenvalues of vibrating membranes, Proc. London Math. Soc. 11 (1961), 419-433. MR0129219 (23:B2256)

[44] G. Pólya, Sur quelques membranes vibrantes de forme particulière, C. R. Acad. Sci. Paris 243 (1956), 469-471. MR0080461 (18:250a)

[45] G. Pólya, Sur les fréquences propres des membranes vibrantes, C. R. Acad. Sci. Paris 242 (1956), 708-709. MR0074672 (17:628b)

[46] M. H. Protter, Can one hear the shape of a drum? revisited, SIAM Rev. 29 (1987), 185-197. MR889243 (88g:58185)

[47] Yu. Safarov, Fourier Tauberian Theorems and Applications, J. Funct. Anal. 185 (2001), 111-128. MR1853753 (2002j:46047)

[48] Yu. Safarov, Lower bounds for the generalized counting function in The Maz'ya anniversary collection, vol. 2 (Rostock, 1998), Oper. Theory Adv. Appl., 110, Birkhäuser, Basel, 1999, pp. 275-293. MR1747899 (2001d:47036)

[49] C. J. Thompson, On the ratio of consecutive eigenvalues in $n$ dimensions, Stud. Appl. Math. 48 (1969) 281-283. MR0257592 (41:2242)

[50] H. Urakawa, Lower bounds for the eigenvalues of the fixed vibrating membrane problems Tohoku Math. J. 36 (1984), 185-189. MR742593 (85m:35045) 
[51] H. Weyl, Das asymptotische Verteilungsgesetz der Eigenwerte linearer partieller Differentialgleichungen, Math. Ann. 71 (1911), 441-479.

[52] H. C. Yang, Estimates of the difference between consecutive eigenvalues, 1995 preprint (revision of International Centre for Theoretical Physics preprint IC/91/60, Trieste, Italy, April 1991).

Department of Mathematics, University of Arizona, 617 Santa Rita, Tucson, Arizona 85721

E-mail address: hermi@math.arizona.edu

$U R L$ : http: //www . math.arizona. edu/ hermi 